\title{
Je voi(e)s double(s): L'itinéraire littéraire de Nadine Ltaif
}

\author{
Christl Verduyn
}

\begin{abstract}
I See(s)/Double(s): The Itinerary of Nadine Ltaif ${ }^{1}$
There is a rich body of writing developing in Quebec by women born elsewhere with an(other) cultural experience. One of the constants in this corpus is the importance accorded the act of writing. For it is writing that enables these women to probe the events of migration, uprooting, exile and a consequent ambivalent attitude to both countries, the one of birth and the one of adoption. The question of language is "the ultimate frontier of alterity." In addition, women write of their condition as women, of economic and sexual exploitation, of mother-daughter relations, of madness. Both sets of issues are explored simultaneously in the story of her coming to writing by Nadine Ltaif, an Arabic-speaking Lebanese Quebec woman writer. In the "Metamorphoses of Ishtar," she is primarily concerned with language, specifically with the absence of words and the difficulty this poses for a writer who must assemble a universe of words. The poetic sequence deals with her long adventure in writing through a change in languages from Arabic to French, away from the singular language of Camel, the language of Man, to the language of the Sirens, that is, to the multiple languages of women. The work on language(s) leads her to the knot mer/mêre (sea/mother), explored further in "Between the rivers," where the narrator passes through psychic alienation to find herself woman, (re)discovering herself in Montreal. This discovery is now perceived as an important reason for her having taken the route of migration and exile. In this second book, the narrator returns to the womb of mothers/seas, to the plural languages of osmosis, metamorphosis, transformations. In Montreal, "between the rivers" "I" is reborn at the same time as I gives birth to "She." "I" is shipwrecked, in perpetual movement, without roots or shelter, outside herself - in exile or mad, "under the shadow of Hecate." Exile is both self-division and separation from the mothers/seas. Thrown into the river she is carried toward the "seas/mothers" which are transformed into a Muse, generating writing, a reason to be, here. She comes to realize she has fled to escape from the hatred for her
\end{abstract}


in men's eyes, for in the Arab world, women do not merit a place in which to be a woman. Affirming herself as woman more and more, she finds in the new country a nursemaid or surrogate mother, a language within a language. Her writing is produced under the double sign of woman and plurality, (im)migrant questioning and feminist critique.

Un premier abord à l'écriture des Québécoises qui sont nées ailleurs, ou qui sont nées au Québec mais qui ont une expérience culturelle 'd'ailleurs', permet de relever quelques constantes, soucis en commun, thèmes partagés, ou traits carctéristiques. ${ }^{2}$ Aussi observe-t-on que leurs textes expriment l'importance de l'acte d'écrire. C'est l'écriture qui permet de sonder les expériences de migration, de déracinement et d'exil. Les oeuvres de ces Québécoises explorent l'attitude ambivalente envers le pays d'origine comme envers le pays d'adoption. Elles traitent de la question de la langue - "dernière frontière de l'altérité" comme le dit Régine Robin, qui approfondit l'étude du "phonénomène de l'hétérogène" au Québec. ${ }^{3}$ En témoignant de l'exil, du déracinement, de la nostalgie, de la mémoire, de la langue, les oeuvres de ces écrivaines québécoises participent à l'évolution de l'écriture '(im)migrante' de nos jours. A l'intérieur de cette thématique, elles visent des thèmes plus particuliers à l'écriture de la femme en général. Elles traitent de la condition de la femme, de son identité difficile, de son exploitation économique et sexuelle, du rapport mère-fille, de la folie, etc. La problématique qu'elles explorent relève en même temps du fait d'être femme que du lien à une culture 'différente'. C'est le cas de l'oeuvre de Nadine Ltaif, arabe d'origine libanaise, auteure de deux livres: Les Métamorphoses d'Ishtar (1987) et Entre les fleuves (1991). ${ }^{4}$ Dans son écriture, Ltaif explore simultanément les questions de l'expérience (im)migrante et de l'écriture. Elle traite en même temps de l'exil et du rapport mère-fille, de la différence, et de la langue.

Dans Les Métamorphoses d'Ishtar, la langue apparaît souvent comme un leitmotif. Au sujet de son premier livre, l'auteure a dit qu'elle n'avait pas de vocabulaire:

Le premierjen'avais vraiment pas de vocabulaire ... J'avais très peu de vocabulaire. C'est vrai. Pourquoi? Parce que chaque livre rassemble des mots, un univers de mots. Tu les mets tous ensemble et là, ça fait un livre. Tu n'as pas besoin de tout. Il y a une harmonie qui se crée, il y a des échos sonores qui se répondent, et puis un 
rythme. ${ }^{5}$

Le rythme est important, car, ainsi que l'explique Ltaif, "les Arabes ont le rythme avant d'avoir les mots" (Ishtar, 58). L'auteure embarque sur la "longue aventure" 6 qu'est pour elle l'écriture, et que la fait changer de langue. "Ce que je disais en arabe je le dis maintenant en français," constate le je narratrice de Les Métamorphoses d'Ishtar (61). La question que le texte pose au départ, c'est "comment retrouver le rythme, un rythme autre/ que celui d'une lamentation?" (8).

Les raisons pour se lamenter sont nombreuses: la misère que le je narratrice voit autour d'elle; la destruction de la guerre entre Arabes et Juifs; la faim des foules qui fait qu'elle refuse de manger elle-même; les ruines causées par les bombes. Dans une deuxième partie, Les Métamorphoses d'Ishtar raconte la lamentable "Histoire du chameau," qui décrit les torts de l'Homme (22): "Tout le dossier sur la torture/ toute la lutte africaine contre l'apartheid/ toute l'histoire des disparitions" (14). A cette histoire correspond une langue, celle de l'Homme, que le chameau a apprise. Il y a cependant une autre histoire et une autre langue, celles de la reine de la nuit et du matin. Cette dernière parle une langue de femmes, la langue des Sirènes, ou plutôt des langues de femme, car "mille et une langues ont parlé (1)a langue" que le je retrouve dans la troisième partie de Les Métamorphoses d'Ishtar: "Les Sirènes." Par l'intermédiaire de la quatrième partie "Ishtar" - un seul poème adressé à la déesse ${ }^{7}$ - on fait la transition au présent et à la cinquième et dernière partie du livre: "Fleur de Grenade". C'est la fin d'hiver 1985 à Montréal, et leje "change delangue" tout en gardent ses mots: "Je cherchemes mots comme Ishtar chasse le Tigre ... je fais très attention, et j'appréhende ce lieu où se posent mes pieds" (37). C'est à la langue et aux mots que le je revient en découvrant le nouveau pays, en hésitant à nouveau entre rester et partir. Si elle reste, c'est à cause de l'écriture, "parce qu'il faut ainsi sauver l'écriture des paroles asséchantes du Chameau" (38). Elle s'installe à Montréal et elle écrit.

L'écriture, la langue, les mots mènent à la mère/mer, thème décisif dans l'oeuvre de Nadine Ltaif. Chez cette auteure, remarque Pierre Bertrand dans son essai "La Langue et l'écriture," 8 "la langue perd ses problèmes, oublie ses 'debats' pour ne plus que ressentir, et faire sentir, la douleur, celle de l'exil sans doute, celle d'être séparé de la mer, de la patrie, de la mère." Le thème de la mère/mer est approfondi dans le deuxième livre Entre les fleuves, où Ltaif pousse l'exploration de la langue, ainsi que celle de deux questions posées par l'écriture 'migrante': 
Qui suis-je, et Où suis-je. L'auteure se découvrira profondément femme, et elle se (re)trouvera dans la ville de Montréal. Assumer son être de femme s'avère être une raison importante pour l'exil et l'(im)migration. La nourrice, et d'autres mères symboliques, en est une autre.

\section{Mere/Mer}

Dans Entre les fleuves, Nadine Ltaif rejoint le ventre de la mère/mer, ou "des mères/mers," comme elle le précise, ${ }^{9}$ "puisqu'on parle de langues au pluriel." En effet, le texte suggère qu'il y a plus d'une mère, comme il y a plus d'une langue. Là où le père est "unique,"10 la mère est double, multiple, pluri/elle(s). Elle a plus d'un visage, y compris celui du je narratrice. Entre "Elle" et "Je", il y a osmose, métamorphose. Entre deux fleuves raconte comment Je a été complètement amoureuse $\mathrm{d}^{\prime \prime}$ Elle sous toutes Ses formes, Ses âges"(8). Dans son deuxième livre même plus que dans son premier, Nadine Ltaif traite de métamorphoses, de transformations, de transfigurations. "Elle" a le visage changeant, tout

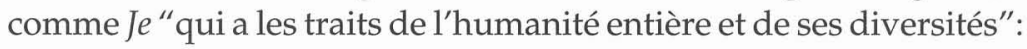

... C'est difficile de me décrire tellement je me vide pour recevoir tant de representations graphiques de la fureur, de la douleur, de la joie en toutes les langues. Je ne sais plus ma langue natale. Tellement j'ai entendu de langues dans mon enfance. (36)

Se nommant Melaina Kole, Je explique que la mélancolie est sa 'raison d'être' dans L'île de M.(ontréal) - "Ile Magique", "colline ensorcelée", "terre d'exil": "Ma terre à moi. Promise. Non. Insensée. Parce qu'elle est humaine. J'ai élu pour terre son corps à Elle. Élu pour territoire d'où naître une deuxième fois. De personne" (26). A Montréal, situé "entre les fleuves," Je renaît en même temps qu'elle donne naissance - à Elle. "Me voilà renaître à nouveau entre ses doigts ... je décidais qu'Elle allait naître de moi aussi" (7). Le rapport mère-fille est complexe et baigne "dans une eau trouble" (16). Je perd son équilibre, et fait naufrage. Dans "l'île du naufragé", "rue du coeur", elle délire, "effrayée d'être en exil" (8), "en mouvement perpétuel. Sans racines. Sans abris. La tempête elle même. Comme une épreuve. Ou bien comme une folie. Je ne me calmais pas. Rien ne me calmait. Je n'étais pas moi. Lieu vacant, réceptacle" (9). Voici l'expérience navrante d'Entre les Fleuves, où Je se trouve, ainsi que le soulignent le titre des première et deuxième parties, en exil et "à l'ombre d'Hécate." Cette déesse "au visage changeant de celle qui aime" s'est 
installée au fond du Je, à la place de son coeur, "Voici mon exil," écrit Ltaif:

Celui qui ne fuit pas seulement la guerre.

Celui qui prend racine aux racines du déracinement profond qui se trouve à l'origine du sens de la vie en dedans de nous.

Qui pose la question de l'origine.

Qui ne trouve pas de réponse.

Qui le sait d'avance par intuition.

Qui ne fait plus la différence.

Pourquoi faire la différence?

Mais qui pose la grande question de la différence entre cultures

entre moeurs et coutumes

entre mourir d'amour et mourir de vivre.

De savoir vivre

avec les autres

de ne plus savoir vivre.

Qui pose la question de l'indifférence

ou de cette différence d'éthique

Je ne sais plus. J'avoue. Plus j'écris moins

je sais. (28)

Dans l'oeuvre de Nadine Ltaif, l'exil prend le sens profond de la séparation à l'intérieur de soi et de la séparation d'avec la mer/mère. "Une loi existe," constate l'auteure, "et c'est la loi de la séparation originelle des cellules. Des cellules se séparent pour donner vie" (50). Séparée de la mer/mère, symboles et sources de vie, c'est à nouveau la mort que $\mathrm{Je}$ confronte. Le texte exprime le désir de mourir, de se laisser aller, entraînée par "la rivière du rêve", emportée 'entre les fleuves': "Loin de ma famille, de mes amies,/ de mon ancienne vie./ Ne réclamant plus d'aucune tribu. /Mourir libre et déracinée" (32).

\section{Femmes}

Mais les fleuves, on le sait, (ra)mènent à la mer/mère. Dans la troisième partie d'Entre les fleuves, Je se retrouve dans le monde de la mer/mère transformée en Muse. L'écriture est restée possible, voire même nécessaire. Elle ramène à la vie et à la raison. "Quand j'écris je 
rejoins cette vie antérieure d'où je viens. Où je suis née une première fois. Il n'y a pas d'indifférence mais équilibre naturel" (37). Ecrire offre une "raison d'être" ici, plutôt qu'ailleurs. C'est ce qu'affirme la dernière partie d'Entre les fleuves: "Le Langage des Sirènes."

Le langage des Sirènes sert à exprimer le sens profond du Je d'être femme: "Du fond de mon être et dans tout ce que je fais, jusque dans la plus fine ramification de mon être, je serai et resterai une femme" (44). Ce fait se révèle sousjacent à la migration:

Il y aura toujours autour de moi des regards mâles exorbiteés de voir tant de féminité s'exprimer sans la moindre gêne. C'est pour fuir la haine de ces regards que j'ai quitté mon pays ( ... ) Aucune femmen'a la place qu'elle mérite dans les pays arabes d'où je viens. D'où je descends" (44-45).

Dans Entre les fleuves, Nadine Ltaif affirme sa place dans le monde des femmes. Elle s'inscrit dans "l'histoire des femmes à travers le monde," histoire qui est la sienne (48). L'auteure explique qu' "il ne faut pas voir non plus que ce texte est très triste. C'est juste que ... quand on fait une séparation, quand on fait un deuil. On quitte son pays et on a une longue période de ... de deuil ... de mélancolie. Mais comme dit Starobinsky, quand tu passes de l'état de la mélancolie à l'état de fureur, quand tu le dépasses, à ce moment-là, tu arrives à un état d'affirmation, d'intégration ... tu milites ... tu deviens de plus en plus ... femme."11

Dans l'ecriture de Nadine Ltaif, la femme/mère est priviligiée. Ce qui relève de $1^{\prime}$ homme/père (biologique ou symbolique ${ }^{12}$ ) est rejeté; l'univers littéraire de l'auteure se revêt d'une symbolique riche provenant du lien mère/mer. Dans un cas (mère) comme dans l'autre (mer), la mer/mère est nourrice chez Ltaif. C'est surtout pour elle-pour la nourrice - que l'auteure est partie. "Je ne voulais pas la voir mourir," explique Ltaif; "je ne voulais pas la voir crever."13

Le thème de la nourrice se manifeste dès le premier livre de Ltaif. Pierre Bertrand le note, relevant la présence de la "mère surérogatoire qui joue déjà comme une langue dans une autre langue." Le thème réapparaît dans Entrelesfleuves où il est approfondi et vient (dé)nouer le texte:

Lorsque nourisson encore, nourris par deux mères, on ne sait vers quel sein se vouer, sevrée par l'une. Aussitôt nourrie par l'autre.

Lorsque l'image de la nourrice a dominé entièrement votre 
vie. A ne plus vouloir me nourrir du tout. Et vouloir me laisser mourir de faim. Lorsqu'une histoire dévore le creux de votre histoire qui dévore une autre histoire qui dévore une autre histoire ... jusqu'à vous perdre.

Lorsque le labyrinthe redevient votre ventre premier. Alors ... Et repliée sur moi. Retrouvant l'oeuf primordial. Je retrouve les ténèbres de la Jarre. Phénicienne alors, ou fille de Carthage. Ma mémoire se déroule d'elle-même et sans résistance aucune jusqu'à ne plus se rompre. (51)

Le thème de la nourrice s'inscrit dans l'écriture de Nadine Ltaif et vient éclairer la vision/voie/voix double de son oeuvre. Car la nourrice a été mère, tout comme la mère est aussi mer. Voilà autant de sources de vie et de nourriture, que le $J e$ réclame en devenant elle-même mère. Traversant la mer pour venir vivre à Montréal, elle (se) donne naissance, et (se) nourrit en écrivant.

Mère/nourrice, mère/mer, mères symboliques. Mères historiques, bibliques, mythologiques aussi. ${ }^{15}$ L'écriture de Nadine Ltaif puise dans le mythique et dans le féminin. Son oeuvre est peuplée de déesses, danseuses, mères, nourrices, soeurs, amies - une pluralité de femmes qui habite l'imaginaire de l'auteure, et qui contraste avec l'unicité du monde tel que l'a fait l'Homme. C'est sous les signes de femme et de pluralité que se réalise l'oeuvre de Nadine Ltaif, dont l'écriture suit un itinéraire de '(Je) (qui) voi(e)s double(s)' pour aller vers un monde de langues, de cultures, et de vision multiples.

\section{Notes}

1. Voir aussi Christl Verduyn. "Nouvelles voies/voix: L'écriture de Nadine Ltaif,"Quebec Studies (à paraître). La présente en est une version remaniée et abrégée. Ces recherches font partie d'un projet de livre sur l'écriture de quelques femmes '(im)migrantes' au Québec et au Canada anglais. L'étude envisage de présenter les oeuvres des auteures à travers a) un essai critique b) une entrevue avec, et c) un extrait de fiction de chaque auteure. Ce projet fait partie de mes recherches depuis dix ans sur l'écriture des femmes.

2. Voir Christl Verduyn, "La Voix féminine de l'altérité québecoise littéraire," dans Hommages à Réjean Robidoux, Ottawa: Les Presses de l'Université d'Ottawa (à paraître). C'est une version écrite d'une communication présentée à l'Association des littératures québecoises et canadienneanglaise (ACQL), Sociétés savantes, mai 1990. Université de Victoria, 
Colombie britannique. Elle présente une liste bibliographique.

3. Voir La Québécoite (Montréal: Québec/Amérique, 1983) et Le Roman mémoriel (Longueil: Préambule, 1989). Dans ce dernier, Robin demande: "Quel français? Quel registre de langage? Quel usage de la langue? Quelle étrangeté de la langue? Quelles inscriptions des autres langues, de l'anglais, du yiddish, de l'hébreu, du russe, etc." (p. 184)

4. L'un et l'autre publiés aux éditions Guernica à Montréal. Il sera également question dans cette étude d'un film auquel Ltaif a participé au moment d'écrire Entre les fleuves. "L'Arbre qui dort rêve à ses racines" (NFB 1992) est un documentaire qui comporte des témoignages de femmes et d'hommes de générations différentes, venus d'ailleurs vivre au Québec. Le film trace en même temps l'amitié entre la réalisatrice Michka Saal, juive d'origine tunisienne, et l'auteure Nadine Ltaif.

5. Dans "l'Arbre qui dort" alors qu'elle fête la parution de son livre en prenant le petit déjeuner avec son amie Michka Saal.

6. Ainsi qu'elle le dit dans "l'Arbre qui dort."

7. Son nom devenu synonyme de déesse, Ishtar réunit les traits caractéristiques de plusieurs déesses, notamment Aphrodite et Vénus.

8. Vice Versa, no. 28, mars/avril 1990.

9. Dans "L'Arbre qui dort."

10. "Aujourd'hui je choisis de tout abandonner, jusqu'à l'héritage de mon père Unique. A la tête d'une horde de soumis. Sous un tel joug n'ai pas plié" (45).

11. Dans "L'Arbre qui dort."

12. Tel le psychanalyste qui paraît dans " $L$ 'Abre qui dort rêve à ses racines." Dans le film, on voit l'auteure chez lui. Elle est visiblement en désarroi. Le sujet est son père. Ltaif est incapable de parler de lui. Elle balbutie, n'achève pas ses phrases, finit par tomber dans le silence. Un peu plus loin dans le film, de nouveau chez le psychanalyste, Ltaif lui tourne le dos en un geste de refus symbolique.

13. Dans "L'Arbre qui dort."

14. Pierre Bertrand, "La langue et l'écriture,"Vice Versa, no. 28 mars/avril 1990.

15. A la fin de Les métamorphoses d'Ishtar, comme au début de "L'Arbre qui dort rêve à ses racines", il est question de Sarah et Agar. 